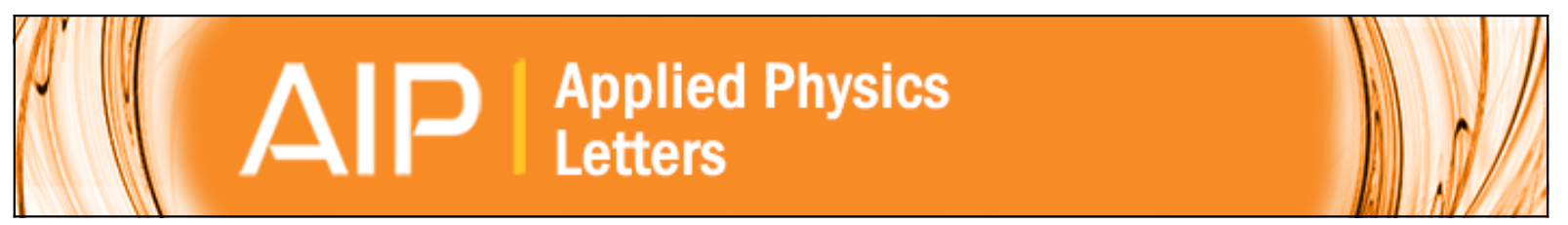

\title{
Bottom-up graphene nanoribbon field-effect transistors
}

Patrick B. Bennett, Zahra Pedramrazi, Ali Madani, Yen-Chia Chen, Dimas G. de Oteyza, Chen Chen, Felix R.

Fischer, Michael F. Crommie, and Jeffrey Bokor

Citation: Applied Physics Letters 103, 253114 (2013); doi: 10.1063/1.4855116

View online: http://dx.doi.org/10.1063/1.4855116

View Table of Contents: http://scitation.aip.org/content/aip/journal/apl/103/25?ver=pdfcov

Published by the AIP Publishing

\section{Articles you may be interested in}

Electronic transport properties of top-gated epitaxial-graphene nanoribbon field-effect transistors on SiC wafers J. Vac. Sci. Technol. B 32, 012202 (2014); 10.1116/1.4861379

Mode space approach for tight-binding transport simulations in graphene nanoribbon field-effect transistors including phonon scattering

J. Appl. Phys. 113, 144506 (2013); 10.1063/1.4800900

A Datta-Das transistor and conductance switch based on a zigzag graphene nanoribbon

J. Appl. Phys. 113, 054304 (2013); 10.1063/1.4790318

Phonon limited transport in graphene nanoribbon field effect transistors using full three dimensional quantum mechanical simulation

J. Appl. Phys. 112, 094505 (2012); 10.1063/1.4764318

Probing transconductance spatial variations in graphene nanoribbon field-effect transistors using scanning gate microscopy

Appl. Phys. Lett. 100, 033115 (2012); 10.1063/1.3678034

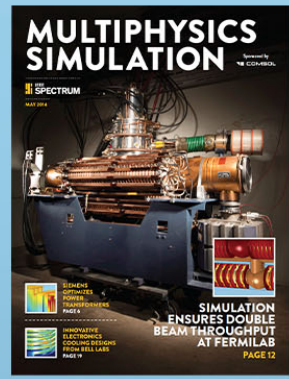

Free online magazine

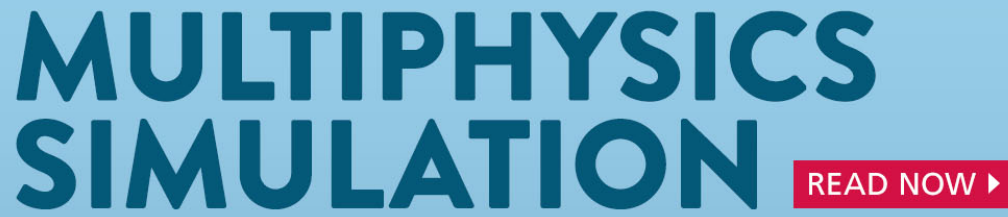




\title{
Bottom-up graphene nanoribbon field-effect transistors
}

\author{
Patrick B. Bennett, ${ }^{1,2}$ Zahra Pedramrazi, ${ }^{3}$ Ali Madani, ${ }^{2}$ Yen-Chia Chen,,${ }^{3,4}$ \\ Dimas G. de Oteyza, ${ }^{3,5}$ Chen Chen, ${ }^{6}$ Felix R. Fischer, ${ }^{4,6}$ Michael F. Crommie, ${ }^{3,4}$ \\ and Jeffrey Bokor ${ }^{2,4, a)}$ \\ ${ }^{1}$ Applied Science and Technology, University of California, Berkeley, California 94720, USA \\ ${ }^{2}$ Department of Electrical Engineering and Computer Sciences, University of California, Berkeley, \\ California 94720, USA \\ ${ }^{3}$ Department of Physics, University of California, Berkeley, California 94720, USA \\ ${ }^{4}$ Materials Sciences Division, Lawrence Berkeley National Laboratories, Berkeley, California 94720, USA \\ ${ }^{5}$ Centro de Física de Materiales CSIC/UPV-EHU-Materials Physics Center, San Sebastián E-20018, Spain \\ ${ }^{6}$ Department of Chemistry, University of California, Berkeley, California 94720, USA
}

(Received 4 November 2013; accepted 1 December 2013; published online 20 December 2013)

\begin{abstract}
Recently developed processes have enabled bottom-up chemical synthesis of graphene nanoribbons (GNRs) with precise atomic structure. These GNRs are ideal candidates for electronic devices because of their uniformity, extremely narrow width below $1 \mathrm{~nm}$, atomically perfect edge structure, and desirable electronic properties. Here, we demonstrate nano-scale chemically synthesized GNR field-effect transistors, made possible by development of a reliable layer transfer process. We observe strong environmental sensitivity and unique transport behavior characteristic of sub-1 nm width GNRs. C 2013 AIP Publishing LLC. [http://dx.doi.org/10.1063/1.4855116]
\end{abstract}

Graphene nanoribbons (GNRs) have been extensively investigated as a promising material for use in high performance, nano-electronic, spintronic, and optoelectronic devices due to their unique physical properties. ${ }^{1-11}$ These properties, however, are critically determined by the precise geometry of the GNR and are degraded by rough edges. Bottom-up, chemical synthesis has been shown to produce GNRs en masse that, unlike GNRs previously studied, possess uniform width and precise edge structure. ${ }^{12}$ Previously, the electronic structure of chemically synthesized GNRs has been studied on their Au growth substrate through Raman, photoemission, and tunneling spectroscopy, ${ }^{12-17}$ but their short length and the metallic growth substrate has thus far prevented standard electronic device fabrication and transport measurements. Here we report layer transfer of chemically synthesized, atomically precise GNRs, enabling study of their physical properties regardless of substrate. Further, we fabricated nano-scale field-effect transistors based on this material and report unique transport behavior characteristic of sub- $1 \mathrm{~nm}$ GNRs.

Growth of GNRs, as previously reported, ${ }^{12}$ occurs via a two step process in which the molecular precursor, 10,10'dibromo-9,9'-bianthryl (DBBA) is thermally sublimed in ultrahigh vacuum (UHV) onto $\mathrm{Au}(111)$, where it is converted into a polymer chain. Thermal cleavage of the labile $\mathrm{C}-\mathrm{Br}$ bonds induces a radical step growth polymerization to yield polymeric GNR precursors. Annealing these polymers on the surface leads to a stepwise cyclization/dehydrogenation sequence yielding fully conjugated GNRs with atomically defined armchair edges. GNRs synthesized with DBBA are exactly 7 carbon atoms across $(\mathrm{n}=7, w=7.4 \AA)$ with a band gap on $\mathrm{Au}(111)$ of approximately $2.5 \mathrm{eV} \cdot{ }^{13-15,18}$

\footnotetext{
a) Author to whom correspondence should be addressed. Electronic Mail jbokor@eecs.berkeley.edu. Present address: 253 Cory Hall MC \#1770, Berkeley, California 94720-1770, USA.
}

Synthesis takes place on the crystalline terraces of clean epitaxial $\mathrm{Au}$ films pre-deposited on cleaved mica substrates. Device fabrication requires the transfer of GNRs to an insulating substrate. Previously, transfer of these GNRs onto $\mathrm{SiO}_{2}$ was reported through mechanical contact of the growth and transfer substrate, ${ }^{12}$ however, we found this method to be irreproducible. We have developed a reliable method of full layer transfer, illustrated in Figs. 1(a) and 1(b). First, poly-methyl methacrylate (PMMA) is spun-cast onto the GNRs and baked $\left(180^{\circ} \mathrm{C}, 10 \mathrm{~min}\right)$ forming a PMMA/GNR/Au/Mica stack. The stack is then floated on concentrated hydrofluoric acid (HF) (40 wt. \%) and occasionally agitated, which induces the mica substrate to delaminate from the $\mathrm{Au}$ growth layer. The PMMA/GNR/Au film is then rinsed twice in water and transferred to Au etchant. Finally, the PMMA/GNR film is rinsed twice again and drawn onto the target substrate surface, $50 \mathrm{~nm}$ thick $\mathrm{SiO}_{2}$ thermally grown on heavily doped silicon in this study. Once the film is adhered to the substrate, it is baked $\left(50^{\circ} \mathrm{C}\right.$ for $5 \mathrm{~min}$ followed by $100^{\circ} \mathrm{C}$ for $5 \mathrm{~min}$ ) to remove residual water and stripped of PMMA with acetone, leaving GNRs on an insulating surface. Raman Spectroscopy performed on samples pre- and post-transfer verifies ribbon integrity is maintained throughout the transfer and device fabrication processes (Fig. 1(c)), confirmed by preservation of the radial breathing like mode $\left(398 \mathrm{~cm}^{-1}\right)$ characteristic for $\mathrm{n}=7$ GNRs. An observed increase in the D peak intensity $\left(1343 \mathrm{~cm}^{-1}\right)$ and slight overall linewidth broadening may be the result of reduced substrate screening effects ${ }^{19}$ or defects induced during transfer. This transfer process is also compatible with any substrate resistant to organic solvents such as acetone.

We next fabricated three terminal transistor devices (Fig. 2(a)). While our GNRs can be as long as $30-40 \mathrm{~nm}$, the average length is $10-15 \mathrm{~nm}$ and so very short physical channel lengths are necessary to contact ribbons at both the source and drain. Therefore, to measure individual ribbons, a large array of source and drain contacts with nano-scale 

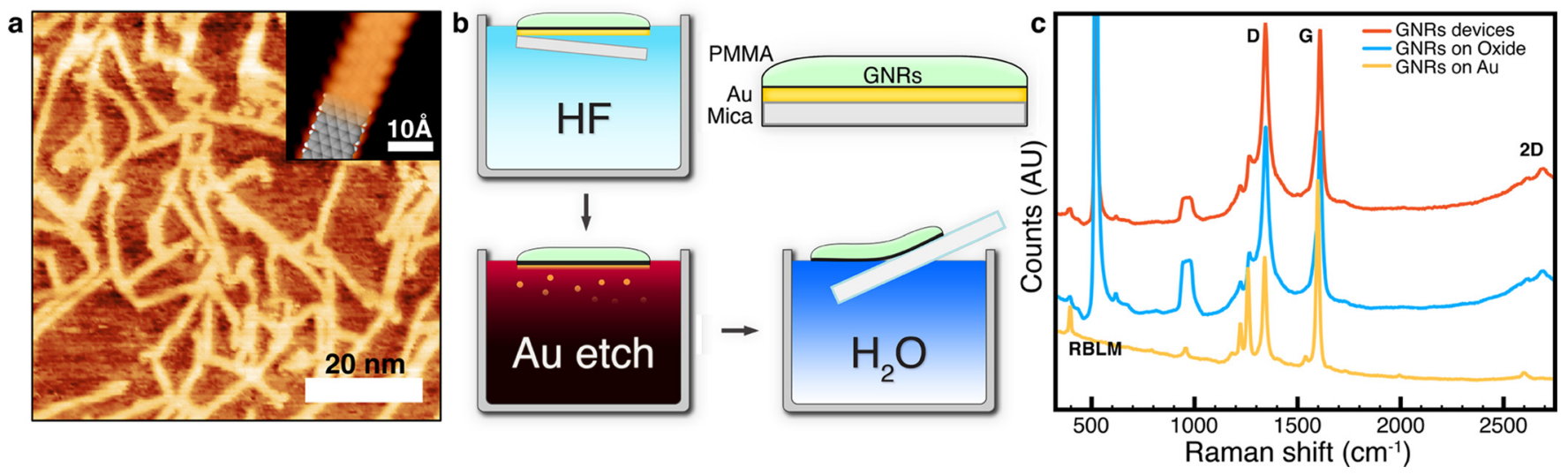

FIG. 1. Growth and transfer of GNRs. (a) Room-temperature STM image of $n=7$ armchair GNRs on their Au growth substrate, tunneling current $I_{t}=0.10$ nA, sample bias $\mathrm{V}_{\mathrm{s}}=1.67 \mathrm{~V}$. Inset: high resolution image of $\mathrm{n}=7 \mathrm{GNR}$ acquired with a low-temperature $\mathrm{STM}\left(\mathrm{T}=7 \mathrm{~K}, \mathrm{I}_{\mathrm{t}}=0.26 \mathrm{nA}, \mathrm{V}_{\mathrm{s}}=-0.40 \mathrm{~V}\right)$. A structural model of the GNR is overlaid on the STM image. For full details regarding STM characterization, see Ref. 18. (b) Illustration of transfer process. The PMMA/GNR/Au/Mica stack is first floated on HF to delaminate the mica substrate. It is then rinsed and placed on Au etchant to dissolve the catalyst layer. It is then rinsed again and pulled onto the target substrate. (c) Raman spectra of GNRs on growth substrate, after transfer on $\mathrm{SiO}_{2}$, and after device fabrication (532 nm excitation wavelength). Peaks characteristic of $\mathrm{n}=7$ GNRs are labeled for reference.

gaps, with $100 \mathrm{~nm}$ widths, were defined using e-beam lithography. First, probe pads (Pd $30 \mathrm{~nm}$ thick) were fabricated via standard liftoff processing using optical lithography and ebeam evaporation. PMMA ( 1 wt. \% $950 \mathrm{~K} \mathrm{M}_{\mathrm{w}}, 4 \mathrm{krpm}$ spin, $180^{\circ} \mathrm{C}$ bake for $10 \mathrm{~min}$ ) was exposed and developed at $-4{ }^{\circ} \mathrm{C}$ in a 7:3 $\mathrm{H}_{2} \mathrm{O}$ :IPA co-solvent solution to pattern individual source/drain contacts. Pd (10 nm thick) was again evaporated and lifted off in acetone. 300 devices were fabricated in total.

Devices were first screened in air and then characterized in a cryogenic probe station. Devices with patterned sourcedrain gaps greater than $30 \mathrm{~nm}$ do not show any conductance, implying that possible inter-ribbon charge transfer between any overlapping ribbons is negligible and that single GNRs did not directly bridge any source-drain gaps this wide. Several devices with smaller gaps between 20 and $30 \mathrm{~nm}$ (14 out of 300 devices with source-drain gaps ranging 20-40 nm) exhibit gate-modulated conductance with oncurrents ranging from tens of $\mathrm{pA}$ to a few $\mathrm{nA}$ at $1 \mathrm{~V}$ sourcedrain bias, $\mathrm{V}_{\mathrm{SD}}$. Because ribbon orientation and position is random, the actual channel length and number of ribbons in each individual device is uncertain. We estimate that in each device there are zero to two GNRs long enough to potentially contact both the source and drain; GNR density is approximately $2 \times 10^{4} / \mu \mathrm{m}^{2}$ with less than $4 \%$ of ribbons longer than $30 \mathrm{~nm}$. Device yield is expected to increase significantly by further reducing the source-drain gap and/or increasing ribbon length during synthesis.

Fig. 2(b) presents electrical characterization of a typical GNR transistor measured in ambient conditions (red) and under vacuum at $77 \mathrm{~K}$ (blue). When measured in air, GNRs contacted with Pd exhibit p-type conduction. Immediately post-fabrication, transistors exhibit large random conductance variations and variable hysteresis due to adsorbed oxygen, water, and residual PMMA on the contact and GNR. ${ }^{20,21}$ Once annealed in vacuum $\left(300^{\circ} \mathrm{C}, 3 \times 10^{-7}\right.$ Torr, followed by a $80^{\circ} \mathrm{C}, 1 \times 10^{-6}$ Torr anneal in the probe station pre-measurement), device behavior switches to n-type conduction, caused by reduction of the contact metal work function due to molecular desorption, ${ }^{22}$ while hysteresis is also greatly reduced by desorption from the channel. About half of devices still display hysteretic ambipolar behavior after vacuum annealing or re-exposure to ambient conditions.

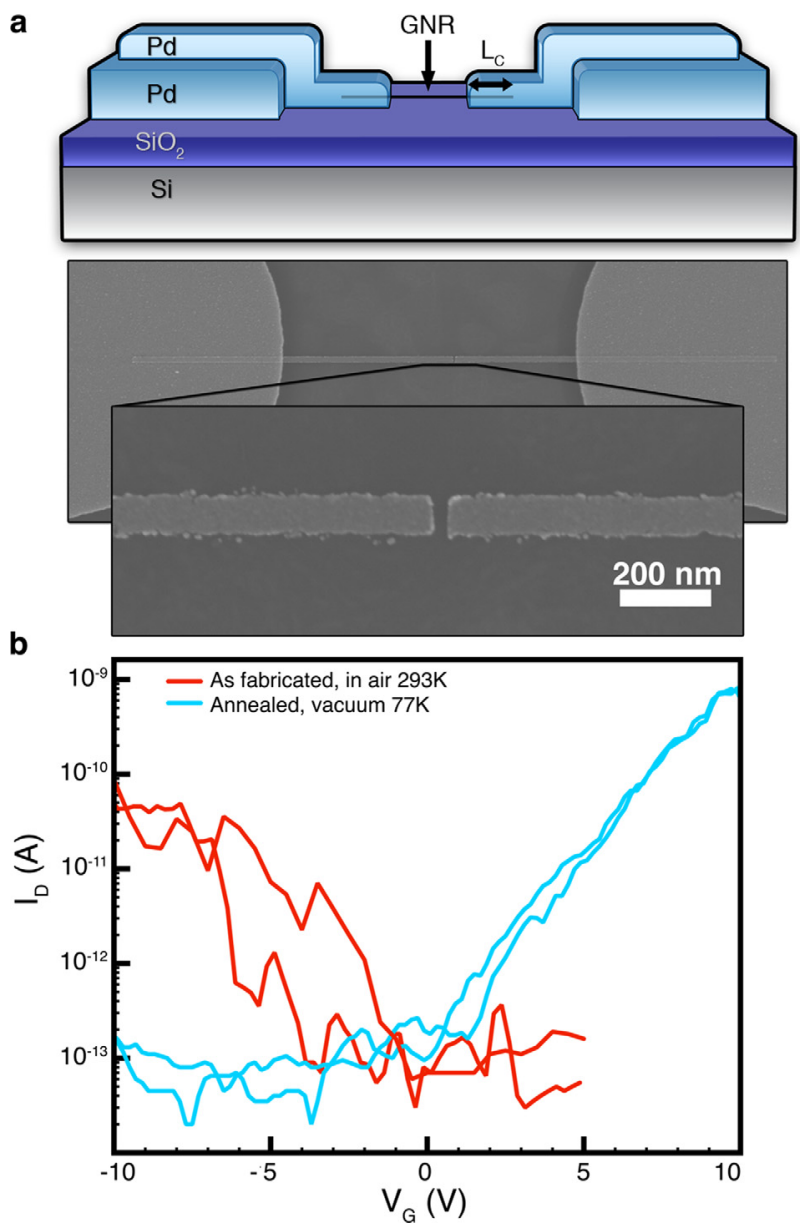

FIG. 2. Device fabrication and environmental behavior. (a) Schematic illustrating device geometry. Because small channel lengths are necessary, a Pd layer forming source and drain contacts to the GNR, using e-beam lithography, is connected to optically defined Pd contact pads. The GNR spans both contacts with some overlap region, $\mathrm{L}_{\mathrm{C}}$, between the GNR and contact. Below: Scanning electron micrograph (1 keV EHT) of the device presented in Fig. 3, $100 \mathrm{~nm}$ wide with a $26 \mathrm{~nm}$ source drain gap. (b) Electrical characterization of a typical device at $\mathrm{V}_{\mathrm{SD}}=1 \mathrm{~V}$ in both air and under vacuum at $77 \mathrm{~K}$. 

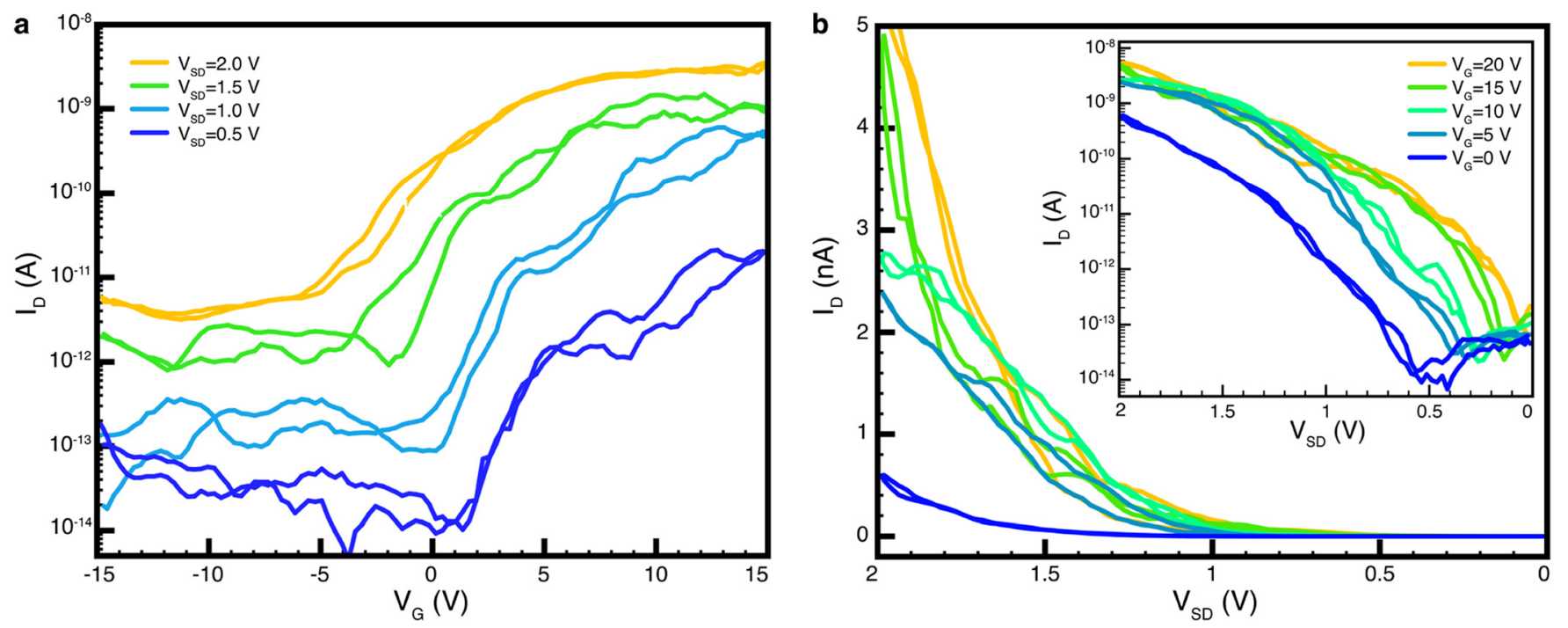

FIG. 3. Electrical characterization of a typical device post passivation, under vacuum, at $77 \mathrm{~K}$. (a) Drain-current response with respect to gate voltage, $I_{D}-V_{G}$, at different source drain bias, $V_{S D}$, and (b) drain-current response with respect to drain voltage, $I_{D}-V_{D}$, of same device at different gate bias, $V_{G}$, inset: The same data presented in logarithmic scale.

Further passivation with a hydrophobic monolayer, hexamethyldisilazane (HMDS), was found to nearly eliminate hysteresis and fully switch device polarity in all devices. Small residual hysteresis effects are attributed to trapped charges within the relatively thick back-gate dielectric and not from molecular adsorbates on the contact or channel. ${ }^{23}$

Transport is largely dominated by the Schottky junction contacts. Full polarity switching through small shifts in contact work function, ${ }^{24}$ relative to the GNR's $\sim 2.5 \mathrm{eV}$ bandgap, suggests that band alignment of the Pd Fermi level falls close to mid-band-gap, a conclusion in agreement with simulations of $n=7$ GNR/Pd interfaces. ${ }^{25}$ Previously published experiments measuring the electrical characteristics of unzipped, chiral Pd contacted GNR transistors 2-20 nm wide, derived from carbon nanotubes (CNTs) through sonochemical exfoliation, observe the presence of a relatively small Schottky barrier at the metal-GNR interface. ${ }^{5,7}$ We see much larger Schottky barriers, potentially as large as $1.25 \mathrm{eV}$, due to the increased band-gap of the much narrower (7.4 ̊) GNRs.

Fig. 3 presents full transport characterization of another typical device, with $26 \mathrm{~nm}$ source-drain gap, as a function of back gate modulation (Fig. 3(a)) and source-drain bias (Fig. 3(b)). High series resistance is also presumed to limit oncurrent in our GNR devices due to the short contact overlap length $\left(\mathrm{L}_{\mathrm{C}}\right)$ between the GNR and the source and drain. Even with our very short channel gaps, contact between the GNRs and Pd is no more than a few to perhaps $10 \mathrm{~nm}$ long depending on GNR length and alignment. Conventional graphene and CNT transistors show large resistance increases as $\mathrm{L}_{\mathrm{C}}$ is decreased past the electron mean free path $(\lambda \sim 200 \mathrm{~nm}),{ }^{26,27}$ suggesting very low transmission probabilities in short contacts for our smooth edged GNRs with low scattering. Despite this, we still see a large measurement limited on-off ratio of $3.6 \times 10^{3}$ at $\mathrm{V}_{\mathrm{SD}}=1 \mathrm{~V}$, clearly demonstrating semiconducting transport in chemically synthesized GNRs.

The observed device behavior is typical of a short channel Schottky barrier device. ${ }^{28}$ In the off-state, leakage is caused by holes tunneling through the drain barrier, which is therefore relatively temperature independent but strongly dependent on $\mathrm{V}_{\mathrm{SD}}$, as larger biases will narrow the width of the Schottky barrier substantially. Also strongly dependent on $\mathrm{V}_{\mathrm{SD}}$ is the threshold voltage $\left(\mathrm{V}_{\mathrm{T}}\right)$ for turn-on, becoming negative for $\mathrm{V}_{\mathrm{SD}}>1 \mathrm{~V}$, due to the strong coupling of the channel to the drain that the gate has to counteract for the device to remain off. The large electric field between the source and drain when the devices are gated on or aggressively biased is sufficient to induce tunneling through the barriers, causing field emission to dominate current flow. This results in unsaturated, nearly exponentially increasing on-current (Fig. 3(b), inset), even at large $\mathrm{V}_{\mathrm{SD}}$, as the barrier continues to narrow and tunneling increases. From this, we can conclude that the resistance of the GNR channel is much lower than the Schottky barrier series resistance, but intrinsic GNR transport properties cannot be observed until these extrinsic factors are ameliorated.

Lowering of the contact work function would reduce the source conduction band barrier height and correspondingly increase the drain valence band barrier, resulting in both improved on- and off-state performance. Further improvement in band alignment should also arise through the use of wider GNRs such as those recently synthesized via similar methods with $1.4 \mathrm{~nm}$ width and $\sim 1.4 \mathrm{eV}$ band-gap. ${ }^{18}$ These are expected to show improved characteristics in a given device due to smaller Schottky barriers and lower effective mass that result from their smaller band-gap. Longer GNRs, through growth optimization, may also reduce contact resistance by increasing $\mathrm{L}_{\mathrm{C}}$.

The narrow width, chemically synthesized GNRs studied here appear to be more sensitive to their environment compared to graphene, CNTs, or significantly wider GNRs previously studied, possibly a consequence of a higher proportion of the exposed, current carrying edge region, ${ }^{14,18}$ relative to the chemically inert surface. ${ }^{29}$ Sensors with greater sensitivity than seen with graphene or CNTs might be achieved through GNR edge modification. Similarly, artificially induced edge states in graphene have been shown to be beneficial to graphene-metal contacts ${ }^{30}$ and may also be 
engineered to enhance GNR-metal electronic coupling. Electronic behavior might also be adjusted through local environment and edge modification in addition to precursor selection during synthesis.

By developing a method for layer transfer of chemically synthesized GNRs we have gained the ability to directly study, using techniques previously unavailable, the behavior of this bottom-up engineered, self-assembled electronic material. In addition to electronic transport measurements, other experiments using chemically synthesized GNRs are also now possible, including optoelectronic and spintronic studies, optical fluorescence measurements, or transmission electron microscopy of freestanding GNRs suspended over patterned membranes. This work highlights the materials development path toward future electronic devices with low series resistance and high intrinsic mobility expected of chemically synthesized GNRs with atomically smooth edges.

Research was supported by the Office of Naval Research BRC Program, by the Helios Solar Energy Research Center, which is supported by the Director, Office of Science, Office of Basic Energy Sciences of the U.S. Department of Energy under Contract No. DE-AC0205CH11231, and by National Science Foundation award DMR-1206512. Work at the Molecular Foundry was supported by the Office of Science, Office of Basic Energy Sciences, of the U.S. Department of Energy under Contract No. DE-AC02-05CH11231. All devices were fabricated in the UC Berkeley Nanolab. We would like to thank Professor M. Lundstrom, Professor Sumon Datta, Dr. D. Haberer, and Professor S. J. Choi for useful discussions.

${ }^{1}$ K. Nakada, M. Fujita, G. Dresselhaus, and M. S. Dresselhaus, Phys. Rev. B 54, 17954 (1996).

${ }^{2}$ M. Y. Han, B. Özyilmaz, Y. Zhang, and P. Kim, Phys. Rev. Lett. 98, 206805 (2007)

${ }^{3}$ Z. Chen, Y.-M. Lin, M. J. Rooks, and P. Avouris, Physica E 40, 228 (2007).

${ }^{4}$ Y.-W. Son, M. L. Cohen, and S. G. Louie, Phys. Rev. Lett.97, 216803 (2006).

${ }^{5}$ X. Li, X. Wang, L. Zhang, S. Lee, and H. Dai, Science 319, 1229 (2008).
${ }^{6}$ G. Liang, N. Neophytou, D. E. Nikonov, and M. S. Lundstrom, IEEE Trans. Electron Devices 54, 677 (2007).

${ }^{7}$ X. Wang, Y. Ouyang, X. Li, H. Wang, J. Guo, and H. Dai, Phys. Rev. Lett. 100, 206803 (2008).

${ }^{8}$ H. Hsu and L. Reichl, Phys. Rev. B 76, 045418 (2007).

${ }^{9}$ J. Guo, D. Gunlycke, and C. T. White, Appl. Phys. Lett. 92, 163109 (2008).

${ }^{10}$ Y.-W. Son, M. L. Cohen, and S. G. Louie, Nature 446, 342 (2007).

${ }^{11}$ W. Y. Kim and K. S. Kim, Nat. Nanotechnol. 3, 408 (2008).

${ }^{12}$ J. Cai, P. Ruffieux, R. Jaafar, M. Bieri, T. Braun, S. Blankenburg, M. Muoth, A. P. Seitsonen, M. Saleh, X. Feng, K. Muellen, and R. Fasel, Nature 466, 470 (2010).

${ }^{13}$ P. Ruffieux, J. Cai, N. C. Plumb, L. Patthey, D. Prezzi, A. Ferretti, E. Molinari, X. Feng, K. Müllen, and C. A. Pignedoli, ACS Nano 6, 6930 (2012).

${ }^{14}$ M. Koch, F. Ample, C. Joachim, and L. Grill, Nat. Nanotechnol. 7, 713 (2012).

${ }^{15}$ S. Linden, D. Zhong, A. Timmer, N. Aghdassi, J. Franke, H. Zhang, X. Feng, K. Müllen, H. Fuchs, L. Chi, and H. Zacharias, Phys. Rev. Lett. 108, 216801 (2012).

${ }^{16}$ J. van der Lit, M. P. Boneschanscher, D. Vanmaekelbergh, M. Ijäs, A. Uppstu, M. Ervasti, A. Harju, P. Liljeroth, and I. Swart, Nat. Commun. 4, 2023 (2013).

${ }^{17}$ H. Huang, D. Wei, J. Sun, S. L. Wong, Y. P. Feng, A. H. C. Neto, and A. T. S. Wee, Sci. Rep. 2, 983 (2012).

${ }^{18}$ Y.-C. Chen, D. G. de Oteyza, Z. Pedramrazi, C. Chen, F. R. Fischer, and M. F. Crommie, ACS Nano 7, 6123 (2013).

${ }^{19}$ A. Jorio, C. Fantini, M. S. Dantas, M. A. Pimenta, A. G. Souza Filho, G. G. Samsonidze, V. W. Brar, G. Dresselhaus, M. S. Dresselhaus, A. K. Swan, M. S. Ünlü, B. B. Goldberg, and R. Saito, Phys. Rev. B 66, 115411 (2002).

${ }^{20}$ W. Kim, A. Javey, O. Vermesh, Q. Wang, Y. Li, and H. Dai, Nano Lett. 3, 193 (2003).

${ }^{21}$ J. Chan, A. Venugopal, A. Pirkle, S. McDonnell, D. Hinojos, C. W. Magnuson, R. S. Ruoff, L. Colombo, R. M. Wallace, and E. M. Vogel, ACS Nano 6, 3224 (2012).

${ }^{22}$ V. Derycke, R. Martel, J. Appenzeller, and P. Avouris, Appl. Phys. Lett. 80, 2773 (2002)

${ }^{23}$ A. D. Franklin, G. S. Tulevski, S.-J. Han, D. Shahrjerdi, Q. Cao, H.-Y. Chen, H.-S. P. Wong, and W. Haensch, ACS Nano 6, 1109 (2012).

${ }^{24}$ S. Heinze, J. Tersoff, R. Martel, V. Derycke, J. Appenzeller, and P. Avouris, Phys. Rev. Lett. 89, 106801 (2002).

${ }^{25}$ C. Archambault and A. Rochefort, ACS Nano 7, 5414 (2013).

${ }^{26}$ F. Xia, V. Perebeinos, Y.-M. Lin, Y. Wu, and P. Avouris, Nat. Nanotechnol. 6, 179 (2011).

${ }^{27}$ A. D. Franklin and Z. Chen, Nat. Nanotechnol. 5, 858 (2010).

${ }^{28}$ J. Larson and J. Snyder, IEEE Trans. Electron Devices 53, 1048 (2006).

${ }^{29}$ D. A. Areshkin, D. Gunlycke, and C. T. White, Nano Lett. 7, 204 (2007).

${ }^{30}$ J. T. Smith, A. D. Franklin, D. B. Farmer, and C. D. Dimitrakopoulos, ACS Nano 7, 3661 (2013). 